\title{
An experimental investigation of jet flows through a row of forward expanded holes into a mainstream over a concave surface
}

\author{
I-Chien Lee $^{\mathrm{a}}$, Ping-Hei Chen ${ }^{\mathrm{a}, *}$, M.K. Chyu ${ }^{\mathrm{b}}$ \\ ${ }^{a}$ Department of Mechanical Engineering, National Taiwan University, No.1, Roosevelt Road, Section 4, Taipei 10617, Taiwan \\ ${ }^{\mathrm{b}}$ Department of Mechanical Engineering and Materials Science, University of Pittsburgh, Pittsburgh, PA 15261, USA
}

Received 28 May 2006; received in revised form 13 December 2007; accepted 19 December 2007

\begin{abstract}
This study performed detailed measurements of jet flows through a row of forward expanded holes into a mainstream over a concave surface using digital particle image velocimetry. Each of ejected holes had a streamwise inclined angle of $35^{\circ}$ bounded on a concave surface with constant radius of $382 \mathrm{~mm}$. The spacing of adjacent holes is $1.5 \mathrm{D}$. The density and the momentum flux ratio of the mainstream to the jet flow were 1.0. Results show detailed 2D mean velocity maps on several horizontal and vertical planes and a 3D streamline pattern of jet mean velocity. The streamlines of 3D mean velocity clearly display different flow characteristics of the ejected jet flow along the transverse direction. In addition, the particle trajectory of a ring enclosing an ejected jet above the injection hole was also presented to show movement of jet.
\end{abstract}

(C) 2008 Elsevier Inc. All rights reserved.

Keywords: Particle image velocimetry; Jets into mainstream; CVP; Lift-off effect

\section{Introduction}

Transport phenomena around a jet array injecting into a mainstream have been a subject of extensive study, because they are related to many significant applications. One of the major applications is film cooling of turbine airfoils, which protects the airfoil material and structure against the extremely high-temperature exhaust from the combustor. While film cooling has been the workhorse of turbine cooling for nearly three decades and surface heat transfer data are plenty, the technology today is by no means well developed. This is largely attributable to the fact that the knowledge, as well as the database, toward the flow fields dominated by the interaction between the jet flow and mainstream is insufficient. Reports to date have revealed several unique and complex flow features associated with the interaction between the jet flow and mainstream. Most

\footnotetext{
* Corresponding author.

E-mail address: phchen@ntu.edu.tw (P.-H. Chen).
}

notable features include the so-called counter-rotating vortex pairs (CVP) embedded in the jet [1-4], a shear-layer vortex existing on the windward side of the jet [1], a horseshoe vortex prevailing upstream to the jet [5], and a nearwall wake region downstream of the jet [6]. In an attempt to characterize the CVP related flow, Liscinsky et al. [7] performed a visualization study of a jet flow into the mainstream on various cross-sectional planes. Their study exhibited that a round jet produces CVP of kidney shape for blowing ratios $(M)$ in the range about $1-2$. On a related topic, Burd et al. [8] examined the film hole length and the effects of turbulence intensity for jets with a inclination relative to the protected surface. Thole et al. [9] measured the effect of the hole exit shape on the distribution of mean velocity, turbulence intensity, and turbulent shear stress at a blowing ratio of 1.0. More recently, Garlomagno [10] performed flow visualization and velocity measurement using particle image velocimetry. The test geometry used for this study was a jet injected toward mainstream over a flat surface. 


\begin{tabular}{|c|c|c|c|}
\hline \multicolumn{4}{|c|}{ Nomenclature } \\
\hline$C_{\mathrm{p}}$ & $\begin{array}{l}\text { pressure coefficient of the mainstream, } \\
=\left(P-P_{0}\right) /\left(0.5 \rho U_{p w}^{2}\right)\end{array}$ & $X$ & $\begin{array}{l}\text { Streamwise distance from the upstream leading } \\
\text { edge of the middle hole, }[\mathrm{mm}]\end{array}$ \\
\hline $\begin{array}{l}D \\
d_{\mathrm{P}}\end{array}$ & $\begin{array}{l}\text { injection hole diameter at the inlet, }[\mathrm{m}] \\
\text { diameter of seeding particles, }[\mu \mathrm{m}]\end{array}$ & $Y$ & $\begin{array}{l}\text { Transverse distance from the centerline of the } \\
\text { middle hole, }[\mathrm{mm}]\end{array}$ \\
\hline$L_{\mathrm{e}}$ & $\begin{array}{l}\text { eddy length scale of jet flow, was chosen to be } \\
0.5 \mathrm{D}_{\mathrm{p}},[\mathrm{mm}]\end{array}$ & $Z$ & $\begin{array}{l}\text { Vertical distance from the upstream leading } \\
\text { edge of the hole, }[\mathrm{mm}]\end{array}$ \\
\hline$M$ & ratio of momentum flux, $=\rho_{\mathrm{j}} U^{2} / \rho_{m} U_{0}^{2}$ & \multirow{2}{*}{\multicolumn{2}{|c|}{ Greek symbols }} \\
\hline$P$ & static pressure, $[\mathrm{Pa}]$ & & \\
\hline $\operatorname{Re}$ & $\begin{array}{l}\text { Reynolds number based on the diameter of } \\
\text { injection hole, }=\frac{\rho U_{0} D}{\mu}\end{array}$ & $\begin{array}{l}\beta \\
\delta\end{array}$ & $\begin{array}{l}\text { injection angle, }\left[{ }^{\circ}\right] \\
\text { boundary layer thickness, }[\mathrm{mm}]\end{array}$ \\
\hline $\begin{array}{l}S t \\
U\end{array}$ & $\begin{array}{l}\text { Stokes number, }=\frac{\mu}{\mu}=\frac{\tau_{\mathrm{p}} U_{\mathrm{e}}}{L_{\mathrm{e}}} \\
\text { mean velocity, }[\mathrm{m} / \mathrm{s}]\end{array}$ & $\theta$ & $\begin{array}{l}\text { the angle of curved section at the onset of the } \\
\text { bend, }\left[^{\circ}\right]\end{array}$ \\
\hline$U_{\mathrm{e}}$ & $\begin{array}{l}\text { eddy velocity scale of jet flow, was chosen to be } \\
40 \% \text { of the mean velocity, }[\mathrm{m} / \mathrm{s}]\end{array}$ & $\begin{array}{l}\mu \\
\rho\end{array}$ & $\begin{array}{l}\text { dynamic viscosity, }[\mathrm{kg} / \mathrm{m} \mathrm{s}] \\
\text { density, }\left[\mathrm{kg} / \mathrm{m}^{3}\right]\end{array}$ \\
\hline $\begin{array}{l}U_{g} \\
U_{\mathrm{pw}} \\
U_{Y Z}\end{array}$ & $\begin{array}{l}\text { Particle sedimentation velocity, }=\frac{\left(\rho_{\mathrm{P}}-\rho_{\mathrm{a}}\right) d_{\mathrm{p}}^{2} g}{18 \mu}[\mathrm{m} / \mathrm{s}] \\
\text { free stream velocity in the curved section, }[\mathrm{m} / \mathrm{s}] \\
\text { projected velocity component of mean velocity }\end{array}$ & $\tau_{P}$ & $\begin{array}{l}\text { particle relaxation time based on Stokes drag } \\
=\frac{D_{\mathrm{P}} \rho_{\mathrm{P}} C}{18 \mu},[\mathrm{s}]\end{array}$ \\
\hline $\begin{array}{l}U_{0} \\
U\end{array}$ & $\begin{array}{l}=\sqrt{U_{X}^{2}+U_{Y}^{2}}[\mathrm{~m} / \mathrm{s}] \\
\text { the free stream velocity in the straight duct, } \\
{[\mathrm{m} / \mathrm{s}]} \\
\text { velocity fluctuation, }[\mathrm{m} / \mathrm{s}]\end{array}$ & $\begin{array}{l}\mathrm{Su} \\
\mathrm{a} \\
\mathrm{o} \\
\mathrm{P}\end{array}$ & $\begin{array}{l}\text { air } \\
\text { reference point } \\
\text { seeding particles }\end{array}$ \\
\hline
\end{tabular}

It is understandable that both the jet flow and mainstream in a turbine passage experience strong curvature effects, for which the model with a flat surface may not be representative. Goldstein and Stone [11] examined film-cooling performance over curved surfaces and suggested that the phenomenon of coolant jet lift-off, which is detrimental to the film cooling performance, could be greatly affected by surface curvature. As mentioned earlier, in the film cooling literature, studies directed to investigating the detailed flow features and momentum transport with a row of jet injected toward mainstream are rather limited,. Therefore, the primary goal of this study is to characterize the detailed velocity fields in a simulated film cooling setting over a concave wall.

\section{Experimental apparatus and procedures}

Fig. 1a shows a schematic view of the experimental mechanism. The experiments were performed in a wind tunnel with a curved test section. On a concave surface, there were five injection holes through which jets were ejected into the mainstream. A centrifugal blower produced the mainstream of the wind tunnel. Jet flows were supplied by a large storage tank system with a pressure of $8 \mathrm{~kg} / \mathrm{cm}^{2}$. The volume consumption of jet flow at a measured timeperiod of $40 \mathrm{~s}$ was about $1.0 \%$ of the total air storage tank volume. The cross sectional area of the inlet section of the tunnel was $450 \times 450 \mathrm{~mm}$. Downstream of the straight duct, there was a Plexiglas curved duct serving as the test section, and an outlet straight duct. The curved duct had a $135^{\circ}$ bend at a constant radius of $382 \mathrm{~mm}$ over the concave surface. A row of injection holes was located at $34^{\circ}$ from the onset of concave surface. The jet flow was injected through a forward expanded hole with a streamwise inclined angle of $35^{\circ}$ on the concave surface. The lengthto-diameter ratio of the injection hole was 2.2. A schematic view of hole geometry is shown in Fig. 2a. Note that measurements were taken on planes in a Cartesian coordinate system, $X Y Z$, of which the origin was located at the upstream edge of the injection hole, as shown in Fig. 2.

The set-up for DPIV flow field measurements is schematically shown in Fig. 1b. A Nd:YAG pulsed laser (NewWave, Reasearch Minilaser) provided pulsed light sheets at a wavelength of $532 \mathrm{~nm}$. The thickness of pulsed laser sheet is adjusted to approximately $1 \mathrm{~mm}$. Particle images were recorded by a Kodak ES1.0 CCD camera at a resolution of $1008 \times 1016$ pixels. It is necessary to determine the magnification factor of the CCD image compared to the real object on the measured plane. The magnification is measured by a calibration procedure. The calibration procedure employed a grid target (DANTEC, 908X0321) measuring $75 \mathrm{~mm}$ by $95 \mathrm{~mm}$ with an exact dot spacing of $10 \mathrm{~mm}$. The calibration target was aligned with the light sheet at each measured plane before the measurement on the measured plane was executed. A Dantec FlowMap 2500 processor with a buffer memory of $8 \mathrm{~GB}$ was installed in a computer workstation that controlled the synchronized actions between the pulsed laser and the CCD camera.

Two components of the instantaneous velocity vector were measured at six horizontal $X Y$ planes $(Z / D=0.13$, 
a
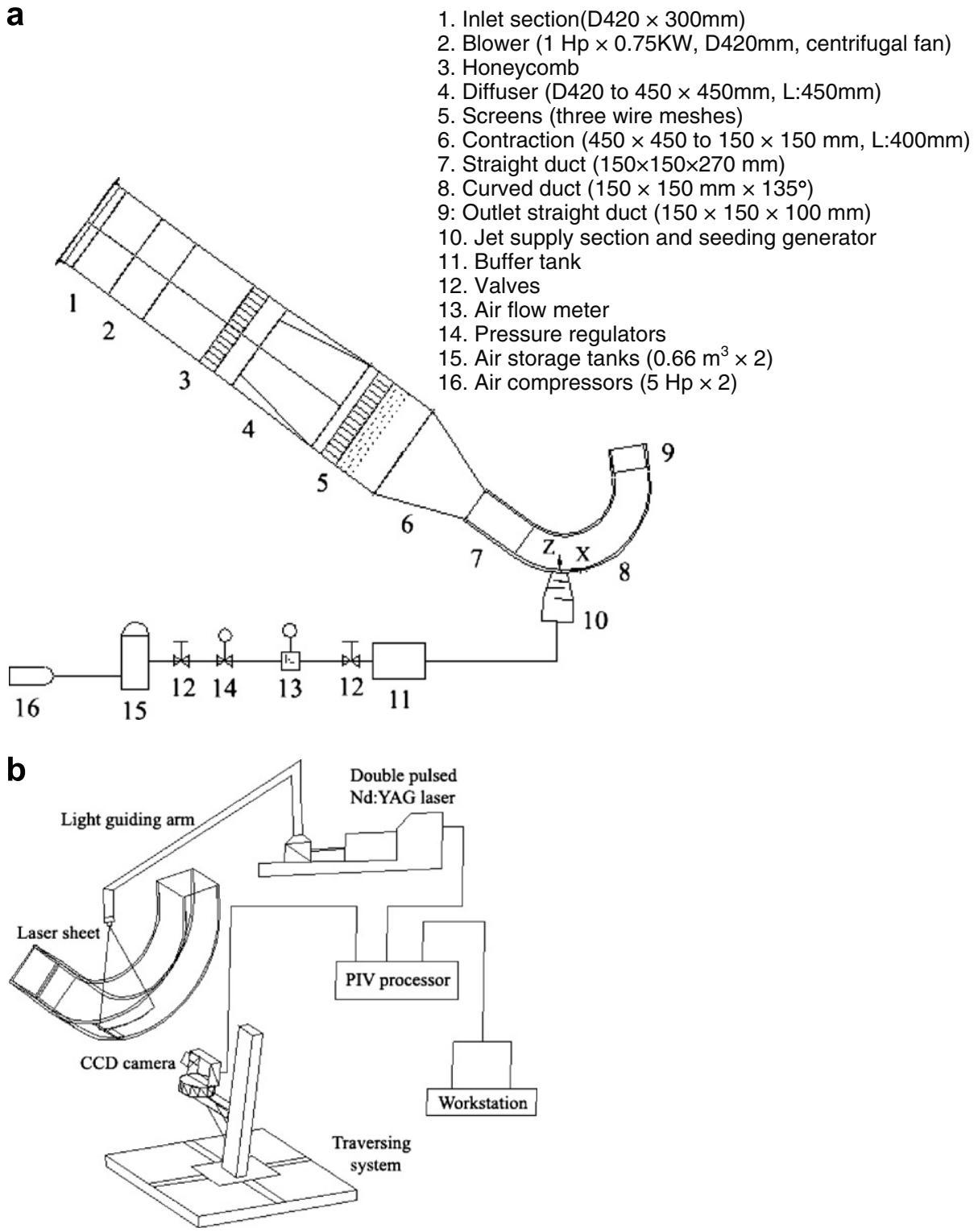

Fig. 1. Schematic views of (a) the experimental set-up, and (b) the DPIV system.

$0.31,0.63,0.88,1.13$, and 1.38 ) and at six vertical $X Z$ planes $(Y / D=0,-0.19,-0.25,-0.45,-0.63$, and $-0.75)$. Since every injection hole was symmetric, the vertical instantaneous velocities were only measured in the negative transverse axis on the vertical $X Z$ plane.

Mainstream velocities were also measured by a hot wire anemometer (Dantec, StreamLine 90C10) with a hot-wire probe (Dantec, 55P63) at a location $200 \mathrm{~mm}$ upstream of the onset of curved duct. Static pressure probes were installed at different locations along the centerline of the concave surface. A pressure transducer (Validyne, DP 103-16) was used to measure static pressure distribution. Pressure signal measurements were converted to digital signals by an AD conversion board (Validyne, UPC 608) for further signal processing.

\section{Operating conditions and experimental uncertainty}

For DPIV measurements, velocity vectors were obtained by calculating the cross-correlation coefficient between two consecutively recorded particle images. Both seeded particle concentration contours and mean velocity vector distributions were acquired at a sampling rate of $15 \mathrm{~Hz}$ and were averaged over 600 instantaneous image pairs. The images were divided into $32 \times 32$ pixel interrogation windows with a $50 \%$ overlap grid to obtain a velocity. The seeded particles were produced by a fog generator using water-based fog liquid [12]. The particle size is about $1-3 \mu \mathrm{m}$ in diameter. If the seeding particles can move with the flow without causing any significant uncertainty, Flagan and Seinfeld [13] showed that the particle's Stokes number, $S t$, must 
a

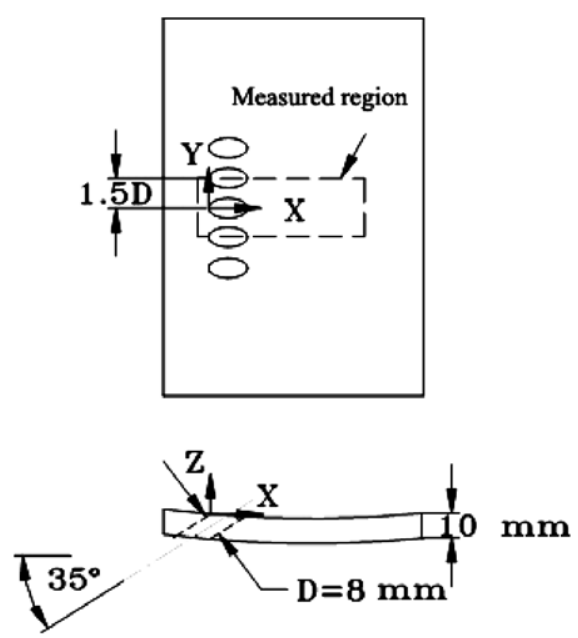

b

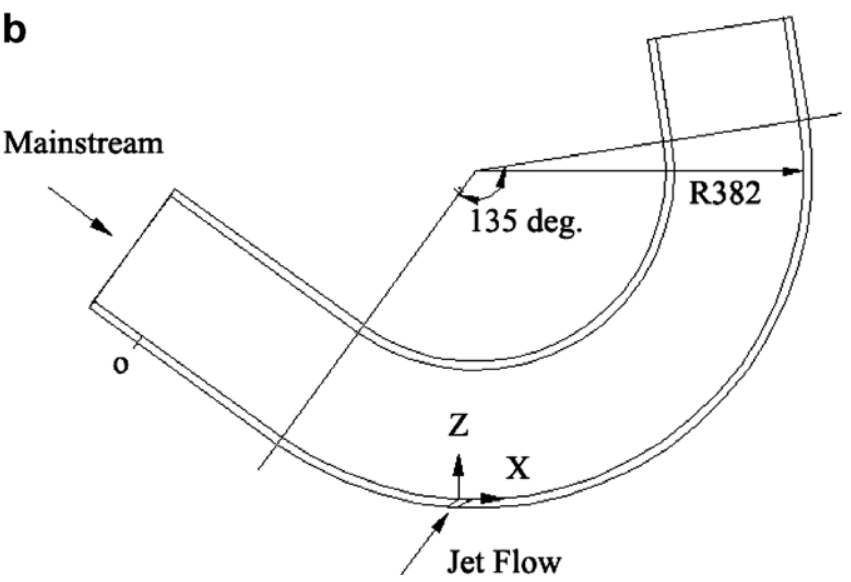

Fig. 2. Schematic views of test region and the Cartesian coordinate system (a) the ejected hole geometry, and (b) the curved duct.

be far smaller than one. In addition, the particle sedimentation velocity [14], $U_{\mathrm{g}}$, of seeding particles must be much smaller than the flow velocity. The particle's Stokes number and particle sedimentation velocity for the worst case in this study are 0.042 and $2.1 \times 10^{-4} \mathrm{~m} / \mathrm{s}$, respectively.

The free-stream velocity, $U_{0}$, of the mainstream in the straight duct was kept at $12.0 \mathrm{~m} / \mathrm{s}$ with a turbulence intensity of $1.5 \%$. Both results were measured at a location $200 \mathrm{~mm}$ upstream of the onset of the curved section. The mainstream Reynolds number was 5820. Both the density ratio and the momentum flux ratio of the mainstream to the jet flow were 1.0. Following the uncertainty analysis of Kline and Mcclintock [15], the uncertainties at a $95 \%$ confidence level were $3 \%$ and $4.5 \%$ for the nominal mean velocity and for the nominal turbulence intensity, respectively. The uncertainty of the seeded particle concentration in the ejected flow was $2.2 \%$. From hotwire measurements; the uncertainties of the mainstream velocity and the mainstream turbulence intensity were $2.0 \%$ and $2.5 \%$, respectively. The uncertainty in the jet mass flow rate was $2.0 \%$. The uncertainty of the pressure coefficient, $C_{\mathrm{p}}$, was $5.6 \%$.

\section{Results and discussion}

\subsection{Mainstream in the curved section}

Since the free-stream velocity on a concave surface of a curved duct may depend on the distance from the wall, this study obtained free-stream velocity from the extrapolated velocity defined by Schultz and Volino [16]. The measured boundary layer thickness, $\delta$, at the onset of the curved section was $6.1 \mathrm{~mm}$ and it gradually increased to $17.0 \mathrm{~mm}$ at the upstream edge of the ejected holes.

Fig. 3 shows the streamwise distribution of pressure coefficient, $C_{\mathrm{p}}$, on the concave surface, which is positioned only at a mainstream without ejected flows. The freestream velocity, $U_{\mathrm{pw}}$, was also calculated from the wall static pressure according to the method proposed by Muck et al. [17]. As observed in Fig. 3, the mainstream decelerated at the onset of the curved section and then maintained a constant velocity in the region where circumferential angle $(\theta)$ ranged from $19^{\circ}$ to $32^{\circ}$ on the concave surface. In the $32^{\circ}<\theta<116^{\circ}$ region, the value of $C_{\mathrm{p}}$ decreases slightly as the mainstream is accelerated. In this study, the value of $U_{\mathrm{pw}}$ was $11.6 \mathrm{~m} / \mathrm{s}$ at the upstream edge of the injection hole. DPIV measurements of the flow field were performed in the region where the value of $X / D$ ranged from -0.5 to 7.0 , corresponding to the circumferential angle ranging from $33.8^{\circ}$ to $60.8^{\circ}$.

\subsection{Mean seeded particle concentrations of the jet flow}

The flow visualization technique was used for quantitative flow field measurement by PIV. Fig. 4 shows PIV timeaveraged jet flow images on a $X-Y$ plane obtained from 600 images at four elevations of $Z / D=0.13,0.31,0.63$, and 0.88 . The dark image indicates the area in which the flow is dominated by the mainstream. As shown in Fig. 4, the jet shape gradually changes from a kidney shape near the hole exit to an oval shape at $Z / D=0.88$. This figure also shows the spanwise periodic behavior of the image, which indicates the uniformity of ejected jets through five injection holes. Fig. 5 shows the mean seeded particle concentration contours at different values of $Z / D$, which can be regarded as flow visualization pictures. Note that the

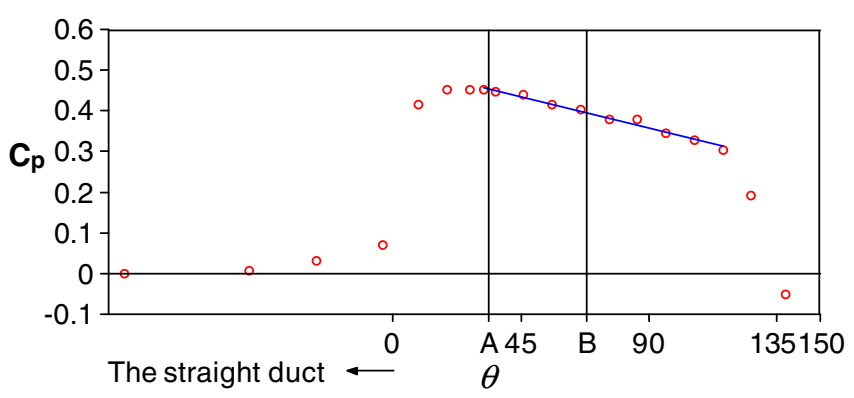

Fig. 3. Streamwise distribution of $C_{\mathrm{p}}$ along the concave wall. (The DPIV measured area lies between $\mathrm{A}$ and $\mathrm{B}$ ). 


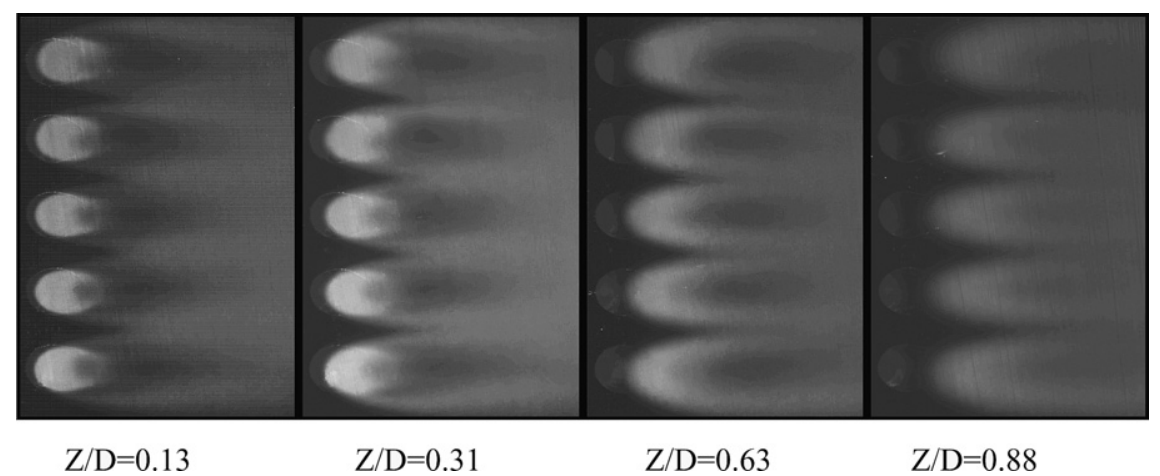

Fig. 4. PIV time-averaged images obtained from 600 images at four positions of the laser sheet.

a
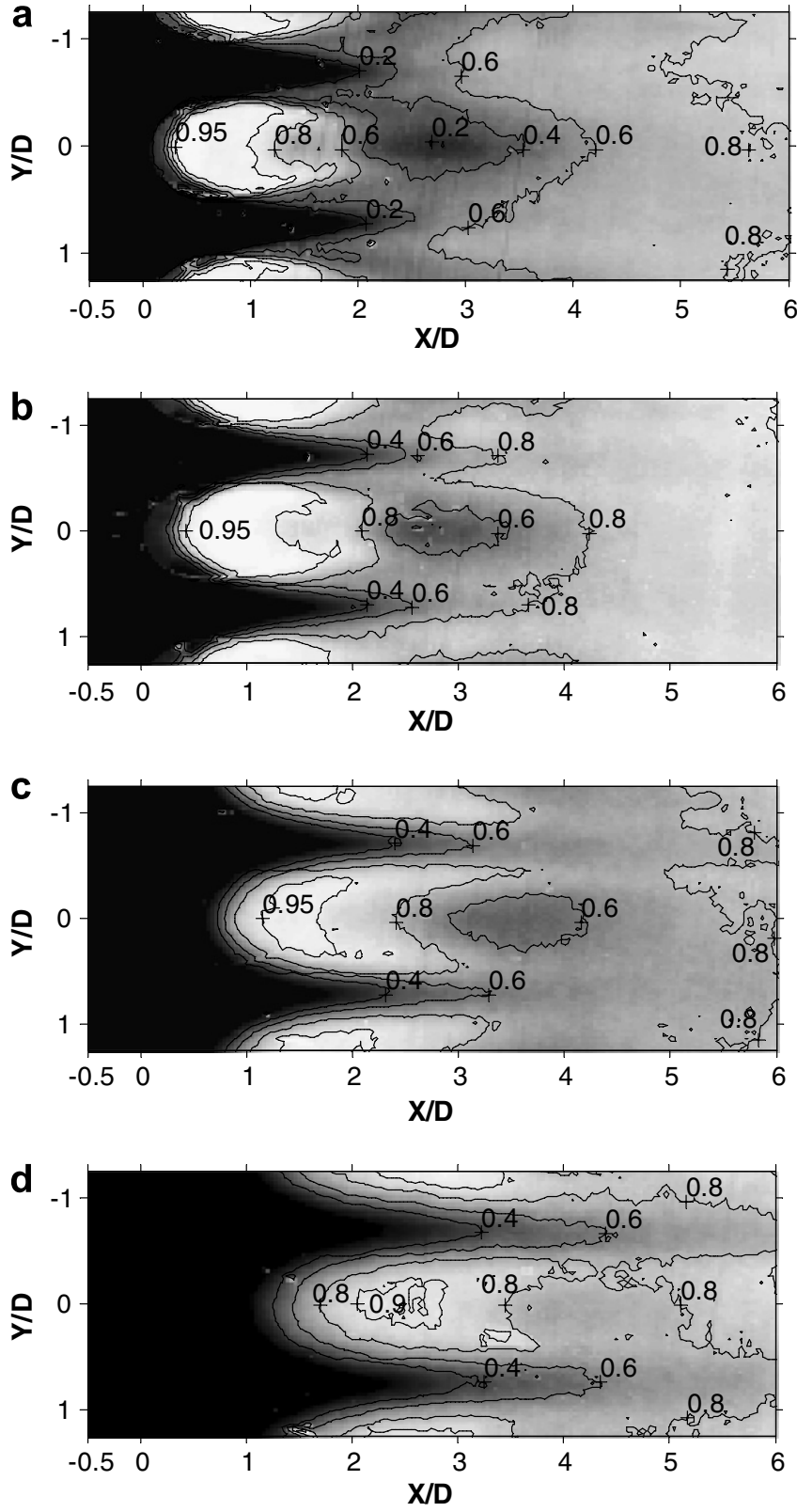

Fig. 5. Contours of mean seeded particle concentration on the different horizontal $X Y$ planes: (a) $Z / D=0.13$, (b) $Z / D=0.31$, (c) $Z / D=0.63$, and (d) $Z / D=0.88$.
CCD images were divided into several interrogation areas $(8 \times 8$ pixels $)$. Each interrogation area corresponds to one measured point. When the seeding particle concentration, $C_{j}$, equals 1.0 at the measured point, the number of pixels containing particle image (the bright spot) is greater than 8 in the chosen interrogation area for all 600 captured images. If the number of pixels having a bright signal is less than 8 , the bright signal is considered background noise and not registered as a particle image. In Fig. 5a at $Z / D=0.13$, the contour of a high mean concentration, $C_{j}=0.95$, shows a clear kidney shape above the ejected hole. There are low particle concentrations behind the ejected hole downstream at this elevation, which means that most of the jet flow moves away from the wall. In Fig. $5 \mathrm{~b}$ at $Z / D=0.31$, the contour of the mean particle concentration at $C_{j}=0.95$ maintains a kidney shape, and the size of kidney shape is larger than that at $Z / D=0.13$. Comparing results between Fig. 5a and b, the expansion of the brightest area indicates jet diffusion in the transverse direction. In Fig. 5c, the area with $C_{j}=0.95$ is reduced as $Z / D$ increases from 0.31 to 0.63 . This is caused by mixing between the jet flow and the mainstream. As observed in Fig. $5 \mathrm{~b}$ and $\mathrm{c}$, the contour of the mean particle concentration, $C_{j}=0.8$, at $Z / D=0.63$ stretches further in the streamwise direction than that at $Z / D=0.31$. This streamwise stretching of the contours is caused by the drag of shear stress exerting on the jet by the mainstream. At a higher elevation of $Z / D=0.88$, a stronger mixing between the mainstream and the jet flow reduces the maximum mean particle concentration to a value of 0.92 and the mean particle concentration gradient in the streamwise direction, as shown in Fig. 5d.

\subsection{Mean velocity field}

Fig. 6 shows three contours of the streamwise component of dimensionless mean velocity, $U_{X} / U_{0}$, at different vertical elevations of $Z / D$. The contour distributions of $U_{X} / U_{0}$ are quite similar in Fig 6a-c, but the maximum contour value increases with vertical elevation away from the wall. At $Z / D=0.13$, low streamwise components of mean jet velocities can be observed on the plane just above the 

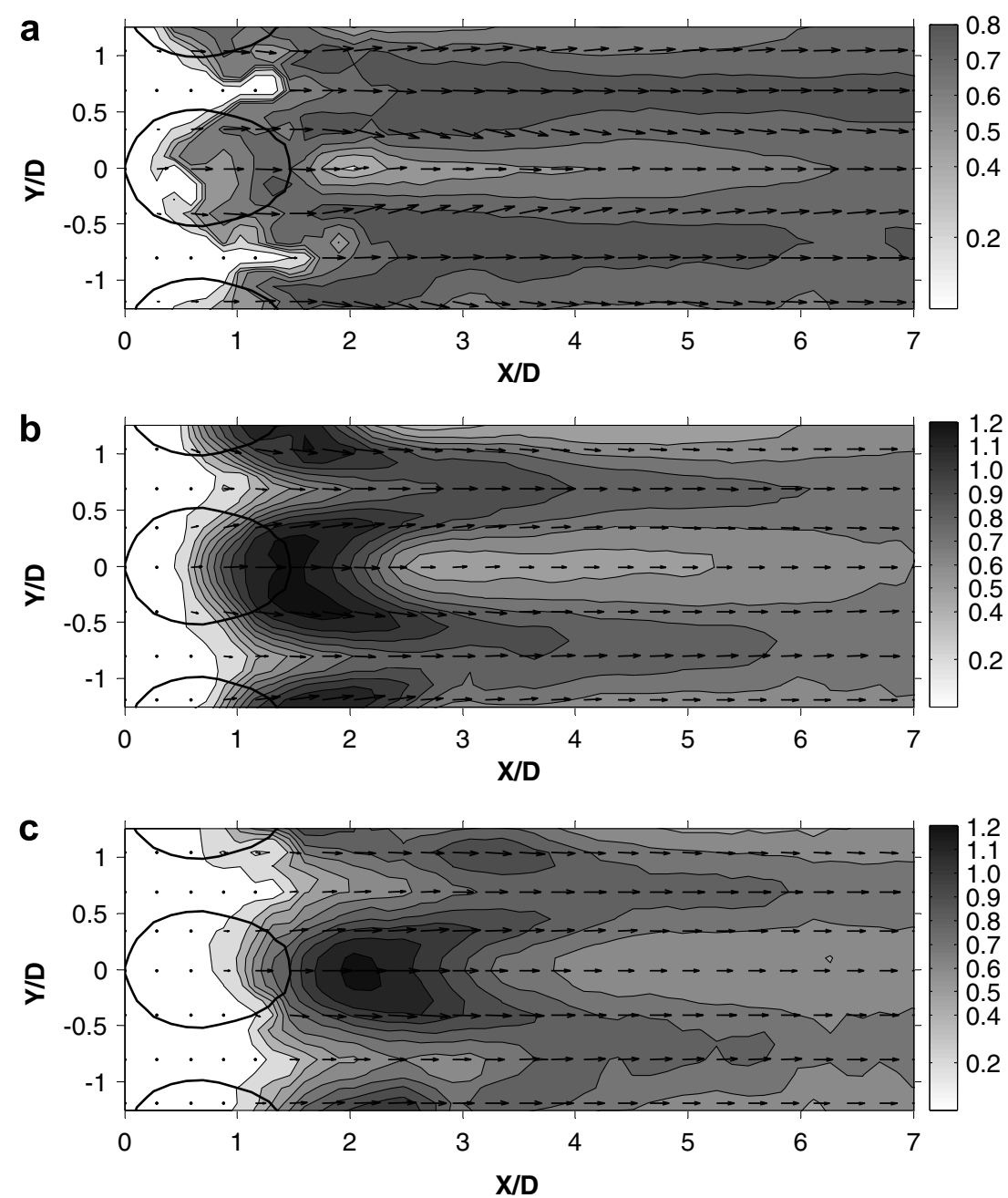

Fig. 6. Contours of streamwise component of dimensionless mean velocity, $U_{X} / U_{0}$ and mean $2 \mathrm{D}$ velocity fields $\left(U_{X}, U_{Y}\right)$ on the different horizontal $X Y$ planes: (a) $Z / D=0.13$, (b) $Z / D=0.63$, and (c) $Z / D=0.88$.

hole exit, particularly in the region just downstream of the injection hole, as shown in Fig. 6a. There is a local minimum in $U_{X} / U_{0}$ at a value of 0.4 in the region just downstream of injection holes around $X / D=2.0$. This indicates that the mainstream is not strong enough to bend the jet flow on the concave surface in this region. In Fig. 6b, the contour of $U_{X} / U_{0}$ on top of the injection hole clearly shows a kidney shape and has a maximum value of 1.2 near the trailing edge of the jet hole as $Z / D$ reaches 0.63 . Apparently, the jet flow in this region is accelerated by jet flow bending due to blockage of the mainstream. However, the maximum value of $U_{X} / U_{0}$ at 1.2 moves further downstream as the measured plane was situated at a higher elevation, $Z / D=0.88$, as shown in Fig. 6c.

Fig. 7 shows transverse component contours of dimensionless mean velocity, $U_{Y} / U_{0}$, at different elevations of $Z / D$. At $Z / D=0.13, U_{Y} / U_{0}$ has an opposite sign but has same magnitude, as shown in Fig. 7a. This symmetric contour pattern indicates that jet flows on both sides of the injection holes move towards the centerline of the hole downstream and the maximum value of $\left|U_{Y} / U_{0}\right| \approx 0.2$ is located near $X / D 2.0-3.3$. There are three almost-parallel lines with the value of $U_{Y} / U_{0}=0$. One is located at the hole centerline and the others are located near the mid-plane between adjacent holes. It is expected that the vortical motion in the jet occurs in the region between these parallel lines. In addition, there is almost no transverse motion in the jet right on top of the injection hole at this elevation.

In Fig. $7 \mathrm{~b}$ at $Z / D=0.31$, the transverse motion in the jet against the hole centerline strengthens in the region directly above the injection hole but weakens in the region downstream. This might indicate CVP already forming in the jet at this elevation. Even higher, at $Z / D=0.63$, the magnitude of $U_{Y} / U_{0}$ in the region directly above the injection hole increases from 0 to 0.15 at $Z / D=0.13$, as shown in Fig. 7c. In addition, the area with $\left|U_{Y} / U_{0}\right| \geqslant 0.1$ at $Z / D=0.63$ is much larger than that at $Z / D=0.31$. Apparently, the strong vortical motion in the jet spreads out from the injection hole to downstream at $Z / D=0.63$.

Fig. 8a-d show vertical component contour plots of dimensionless mean velocity, $U_{z} / U_{0}$, at $Y / D=0.0,-0.25$, 

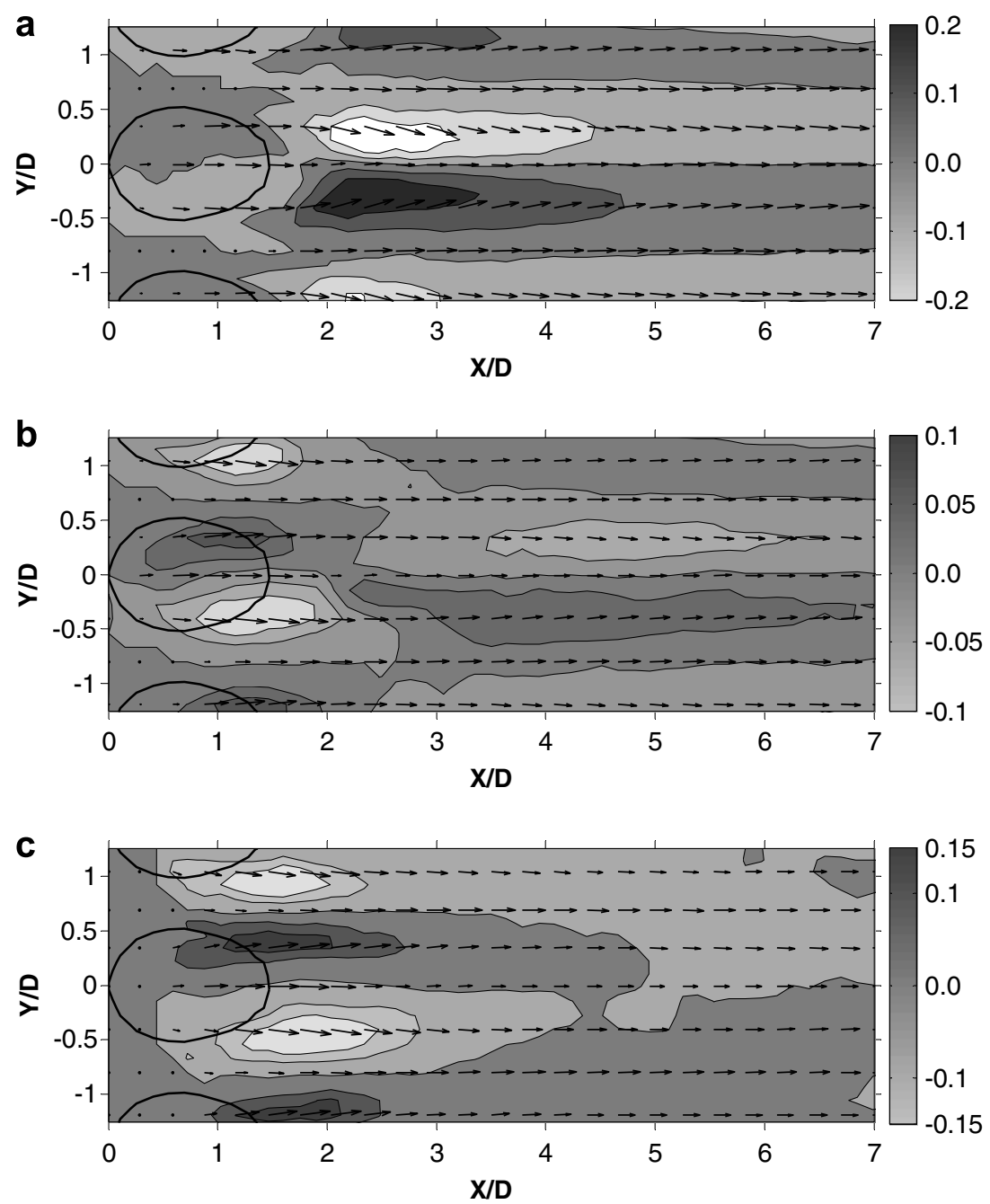

Fig. 7. Contours of the transverse component of dimensionless mean velocity, $U_{Y} / U_{0}$ and mean $2 \mathrm{D}$ velocity fields $\left(U_{X}, U_{Y}\right)$ on the different horizontal $X Y$ planes: (a) $Z / D=0.13$, (b) $Z / D=0.31$, and (c) $Z / D=0.63$.

-0.63 , and -0.75 , respectively. The results in Fig. 8a-b provide evidence that affects the film cooling protection over a concave surface. Downstream of the injection holes, the regions near the wall with positive $U_{z} / U_{0}$ values have poor film cooling protection on the surface. On the center injection hole plane at $Y / D=0.0$, Fig. 8a shows that there are two maxima in the $U_{Z} / U_{0}$ contour plot. One is an absolute maximum located immediately above the hole exit and the other is a local maximum located in the $2.5<X / D<3.0$ region. The latter local maximum in $U_{Z} / U_{0}$ is produced by the impingement of opposite transverse flow motions in the jet moving towards the injection hole centerline. This strong positive vertical velocity downstream of the injection hole provides evidence of the lift-off of jet flow (Goldstein and Stone [11]). Away from the injection hole centerline, the $U_{Z} / U_{0}$ contour plots in Fig. $8 \mathrm{~b}$ at $Y / D=$ -0.25 are similar to those at $Y / D=0.0$, but have lower absolute maximum value in $U_{Z} / U_{0}$ at the upstream edge of the injection hole and no local maximum in $U_{Z} / U_{0}$ in the leeward side of jet flow. Therefore, the lift-off of jet flow near the injection hole centerline is produced by opposite transverse flow motions in the jet near the wall surface. At $Y / D=-0.25$, the jet is bent by the mainstream and is attached to the concave wall surface in the region downstream of the injection hole. At $Y / D=-0.63$, in Fig. 8c, negative values of $U_{z} / U_{0}$ appear in the leeward side of the jet flow. This phenomenon indicates jet flow downwash. A maximum value of $\left|U_{z} / U_{0}\right|$ at 0.35 appears near the concave wall in the $3.2<X / D<4.2$ region. There is no doubt that effective film cooling could be found in this region due to the jet flow downwash. On the midplane between two adjacent holes at $Y / D=-0.75$, the jet flow downwash becomes even more obvious than at $Y / D=$ -0.63 since the maximum value of $\left|U_{z} / U_{0}\right|$ increases from 0.35 at $Y / D=-0.63$ to 0.45 . This is due to interaction between CVP in the neighboring jets. If an observers stands at the upstream side of a row of injection holes to observe the CVP flow direction in the jet, the right CVP vortex 

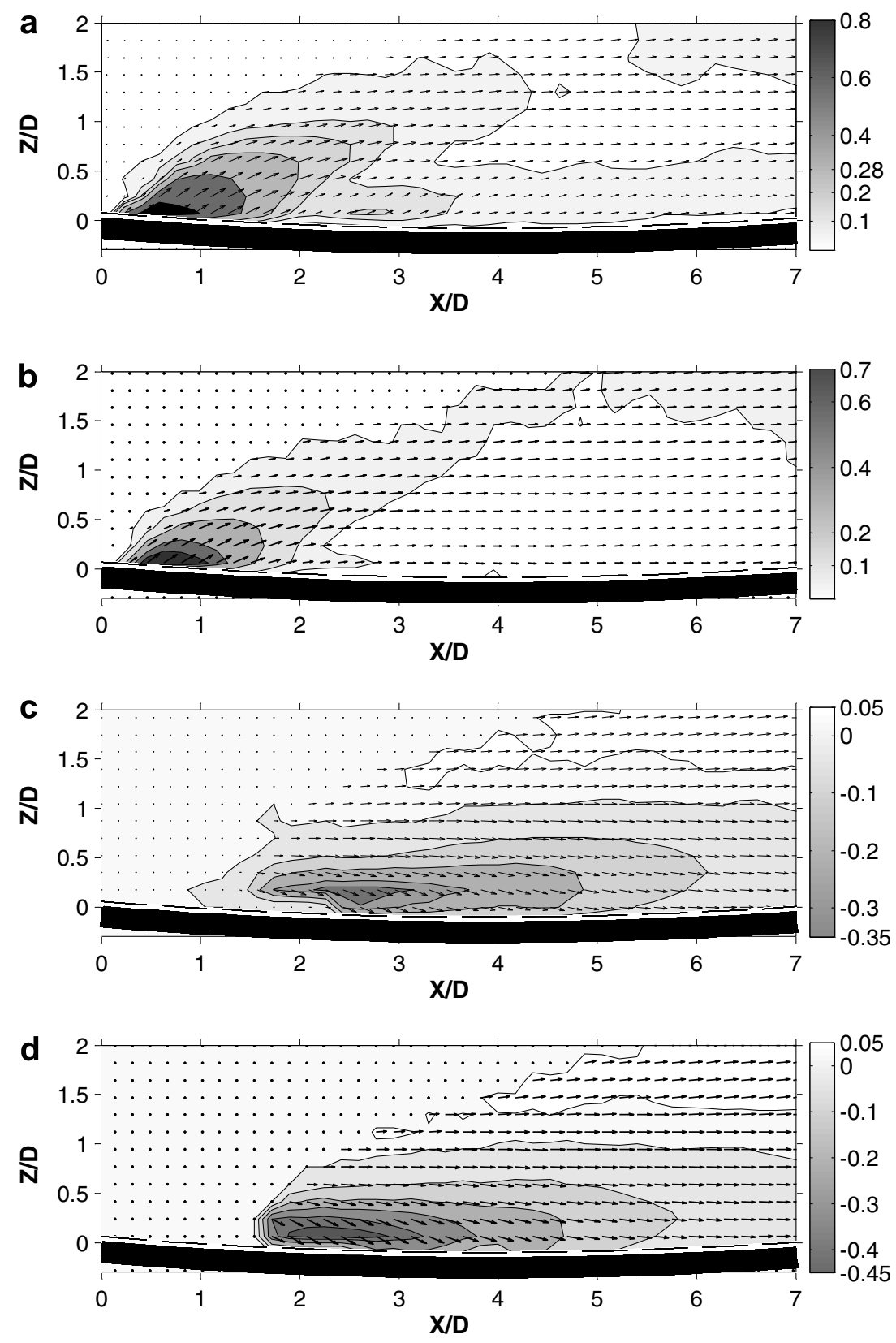

Fig. 8. Contours of the vertical component of dimensionless mean velocity, $U_{z} / U_{0}$ and mean $2 \mathrm{D}$ velocity fields $\left(U_{X}, U_{z}\right)$ on the different vertical $X Z$ planes: (a) $Y / D=0.0$, (b) $Y / D=-0.25$, (c) $Y / D=-0.63$, and (d) $Y / D=-0.75$.

rotates clockwise and the left CVP rotates counter-clockwise. Since the spacing between adjacent holes is only $3 \mathrm{D}$, rotating-vortex pairs in the neighboring jets lie against each other at midplane.

\subsection{Flow patterns of jets into a mainstream}

Fig. 9 shows cross-sectional views of the contour plots of the dimensionless projected velocity component, $U_{Y Z} / U_{0}$, and projected well spaced streamlines at $X / D=$ 2.0 and 3.0, respectively. In Fig. 9a and b, distinct streamline characteristics can be found in two different regions. One region has near straight lines close to the injection hole center plane, and the spacing between neighboring streamlines is narrower near the wall and wider away from the wall. This phenomenon indicates that jet flow upward motion is strongly restrained by the mainstream. The other streamline characteristic shows a strong vortex motion in the left corner. As indicated in Fig. 9a, the vortex has a zero value of $U_{Y Z} / U_{0}$ at location $(Y / D, Z / D)=(-0.45$, 0.33 ) on the plane at $X / D=2.0$. The mainstream blockage (reduction in streamwise velocity component of jet flow in Fig. 6b) and the interaction of adjacent jets (negative vertical velocity component of jet flow as shown in Fig. 8c and d) contributed to the circulation of this streamwise vortex. Jet lift-off can be observed from the upward flow motions 

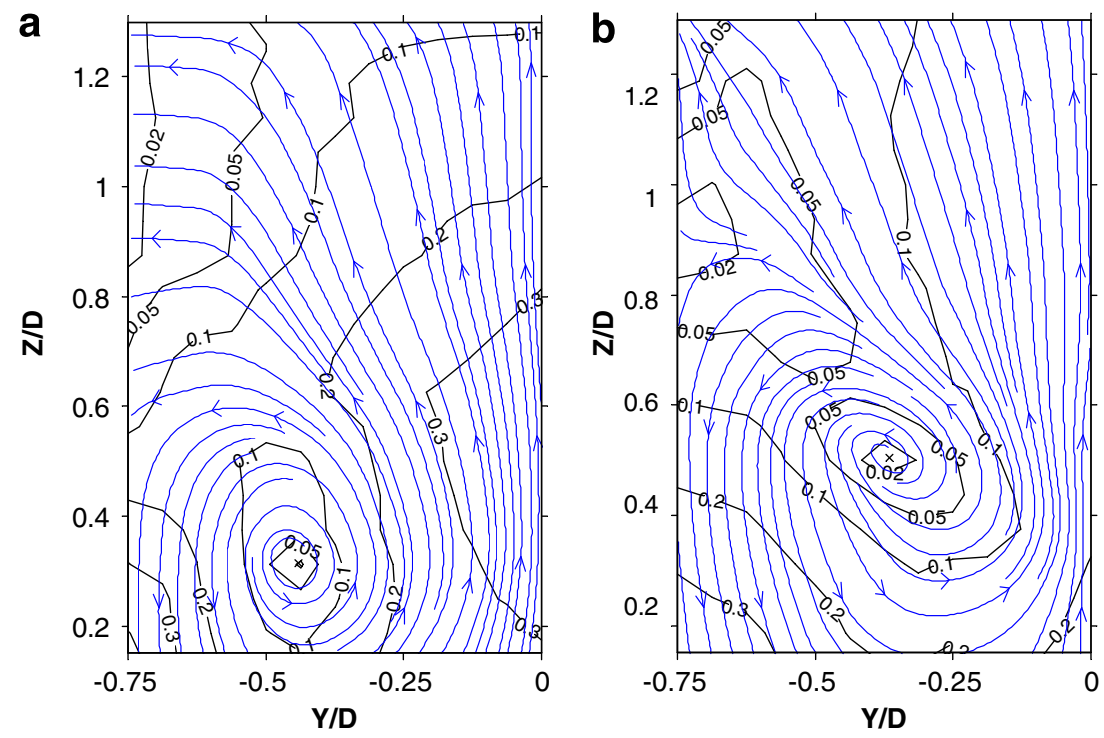

Fig. 9. Cross-sectional views of the $Y Z$ plane streamline distributions with contours of its $2 \mathrm{D}$ dimensionless velocity magnitude $\left(U_{Y Z} / U_{0}=\sqrt{U_{Y}^{2}+U_{Z}^{2}} / U_{0}\right)$ on different streamwise positions: (a) $X / D=2.0$ and, (b) $X / D=3.0$.

in Fig. 9a-b at all streamwise locations. In Fig. 9b, the value of $U_{Y Z}$ near the injection hole center plane decreases from 0.3 to 0.1 as $X / D$ increases from 2.0 to 3.0.

Fig. 10(a)-(d) show the perspective views of streamline distributions where starting points are located at the same vertical elevations of $Z / D=0.19$ on different transverse planes, $Y / D=-0.025, Y / D=-0.25, Y / D=-0.30$, and $Y / D=-0.375$. The construction of three-dimensional streamlines is obtained from the measured time-averaged 3D velocity field using the approach described by Karamcheti [18]. The spacing between starting points along the streamwise direction on each transverse plane is $0.1 \mathrm{D}$. Since the injection angle of jet flow is $35^{\circ}$, no jet flow can be found at an elevation of $Z / D=0.19$ directly above the upstream edge of the injection hole. The upstream most streamline starts at $0.1 \mathrm{D}$ from the upstream injection hole edge. Figs. 11 and 12 illustrate the side and top view of spatial streamline distributions of Fig. 10, respectively. Note that the data shown in Fig. 10a is very much representative for the flow characteristics of the injection center plane. Here, for $Y / D=-0.025$, It is clear that the spacing between jet flow streamlines, remains virtually the same as the jet flow travels downstream. It can be confirmed by results shown in Fig. 11a. In Fig. 10b, jet expansion results in the transverse movement of streamlines starting at $Y / D=-0.25$ towards the midplane of adjacent jets when the jet travels downstream. In addition, if an observer stands at the upstream side of an injection hole row to observe, streamlines are swirled clockwise. As shown in Fig. 10b, streamlines starting from the front portion of the injection hole travel towards the midplane of adjacent jets, but those starting from the rear portion of the injection hole travel upwards and slightly towards the jet hole center plane, as shown in Figs. 11 and 12b. Streamline swirling is caused by CVP in the jet. Streamline swirling becomes more obvious as the transverse streamline starting point moves from $Y / D=-0.25$ to -0.30 , as indicated in Fig. $10 \mathrm{~b}$ and c. Near the transverse side of the injection hole at $Y / D=-0.375$, streamlines starting near the front of the injection hole travel upwards first and then are pushed towards the wall by the mainstream, as shown in Fig. 10d. When the streamlines nearly touch the wall, the present measured data are not close enough to the wall to determine the passage of streamlines. Thus, the streamlines end near $X / D \approx 5.0$, as indicated in Fig. 12d. However, the reason for upstream most streamline termination near $X / D \approx 3.0$ is due to mixing occurring between the mainstream and the ejected jet.

As observed from the streamline passages shown in Figs. 10-12, the jet flow ejected from an inclined jet into a mainstream has different flow patterns depending on various transverse positions. It can be divided into three zones, a straight flow zone, a swirling flow zone, and a touchdown flow zone. Near jet hole center plane (a straight flow zone), the jet flow moves almost straight downstream. As $Y / D$ increases (a swirling flow zone), jet flow swirling becomes more significant. Near the transverse edge of the injection hole (a touch-down flow zone), the reattachment of jet flow ejects can be found, which is caused by blockage of the mainstream.

If one can track the particles on the circular ring enclosing the jet at some locations above the injection hole, one can obtain the physical insight of evolution of ejected jet in the mainstream. Fig. 13 shows the temporal evolution of a ring-shape fluid filament surrounding the ejected jet right above the hole exit. Note that the first ring-type filament right above the injection hole is chosen in such a way that the vorticity of the fluid particle on the ring-type filament remains the same. However, the vorticity of every particle on the filament cannot remain the same as the 
a
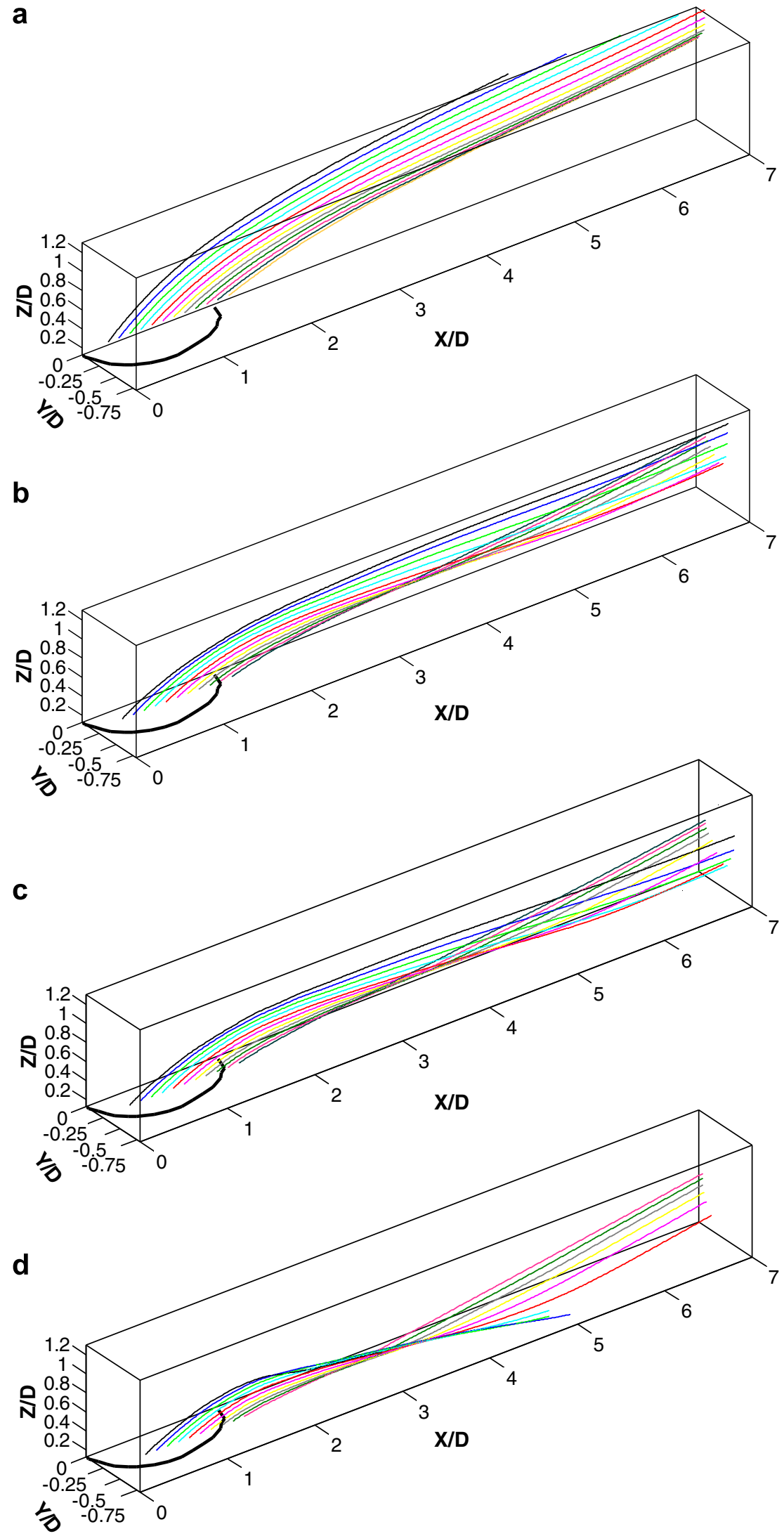

Fig. 10. Perspective views of streamline distributions starting from $Z / D=0.19$ with equal spacing of $0.1 \mathrm{D}$ along streamwise direction at different transverse locations: (a) $Y / D=-0.025$, (b) $Y / D=-0.25$, (c) $Y / D=-0.30$, and (d) $Y / D=-0.375$. 

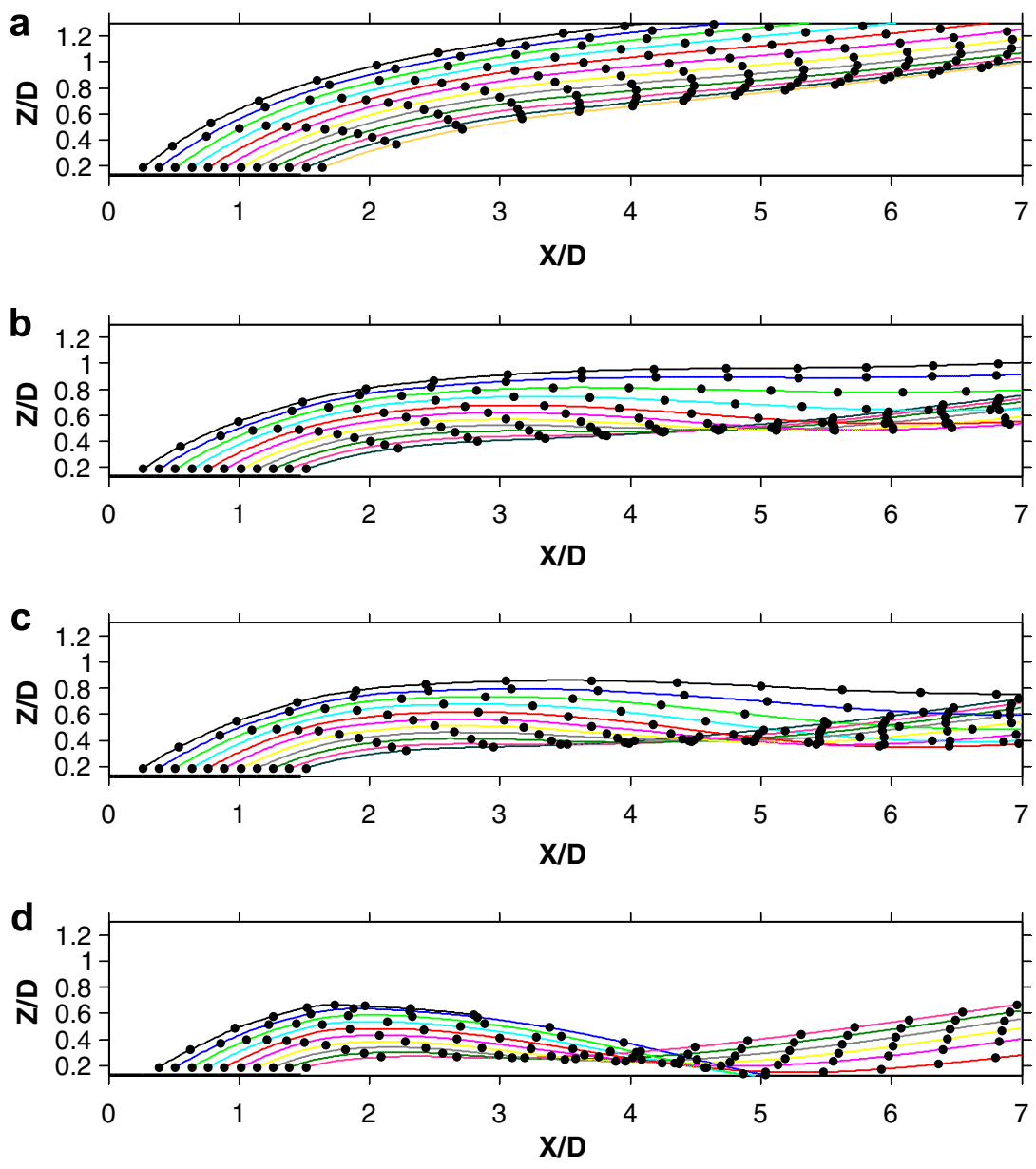

Fig. 11. Side views of streamline distributions of Fig. 11 starting from $Z / D=0.19$ with equal spacing of $0.1 \mathrm{D}$ along the streamwise direction at different transverse locations: (a) $Y / D=-0.025$, (b) $Y / D=-0.25$, (c) $Y / D=-0.30$, and (d) $Y / D=-0.375$. i.e. each dot distance along a streamline keeps a fixed time-period.

ring-shape fluid filament travels downstream. This is because the fluid particle on the filament experiences different levels of the mainstream effect. Since the fluid particles' velocities on the ring are not the same, the ring shape is distorted as the fluid particles move downstream. Successive trajectories of the ring-shape filament in Fig. 13 are constructed by tracing the fluid particles from the first ringshape filament at different times. The upstream portion of the circular ring is tilted up when the fluid particles move downstream because the jet is bent towards the wall by the mainstream. This stretch of circular ring in the transverse direction results from transverse shear stress induced by the interaction between the jet flow, the mainstream, and a pair of streamwise vortices. As mentioned in the previous section, this pair of streamwise vortices occurs in the wake region downstream of the ejected jet.

\section{Conclusions}

This study performed velocity measurements and flow visualizations for a row of inclined jets though a forward expanded hole into a mainstream over a concave surface using a DPIV system at $R e=5820$. Each of ejected holes had a streamwise inclined angle of $35^{\circ}$ bounded on a concave surface with constant radius of $382 \mathrm{~mm}$. the spacing of adjacent holes is $1.5 \mathrm{D}$. The density and the momentum flux ratio of the mainstream to the jet flow were 1.0. The measured region was located downstream of the central injection hole with a streamwise distance, $X / D$, ranging from -0.5 to 7.0. The following conclusions are obtained from measured distributions of mean seeded particle concentrations, three-dimensional time-averaged velocity field, and streamlines of a row of ejected jets into the mainstream:

1. At the center plane, the strong positive vertical mean velocity, $U_{z} / U_{0}$, downstream of the injection hole provides evidence of the jet flow lift-off (Goldstein and Stone [11]). This positive value of $U_{z} / U_{0}$ has poor film cooling protection on the surface. Therefore, jet flow lift-off near the injection hole centerline is produced by opposite transverse flow motions in the jet near the wall surface.

2. A counter-rotating secondary-flow vortex pair immediately forms in the jet at a location between $Z / D=0.13$ and 0.31 directly above the injection hole. 

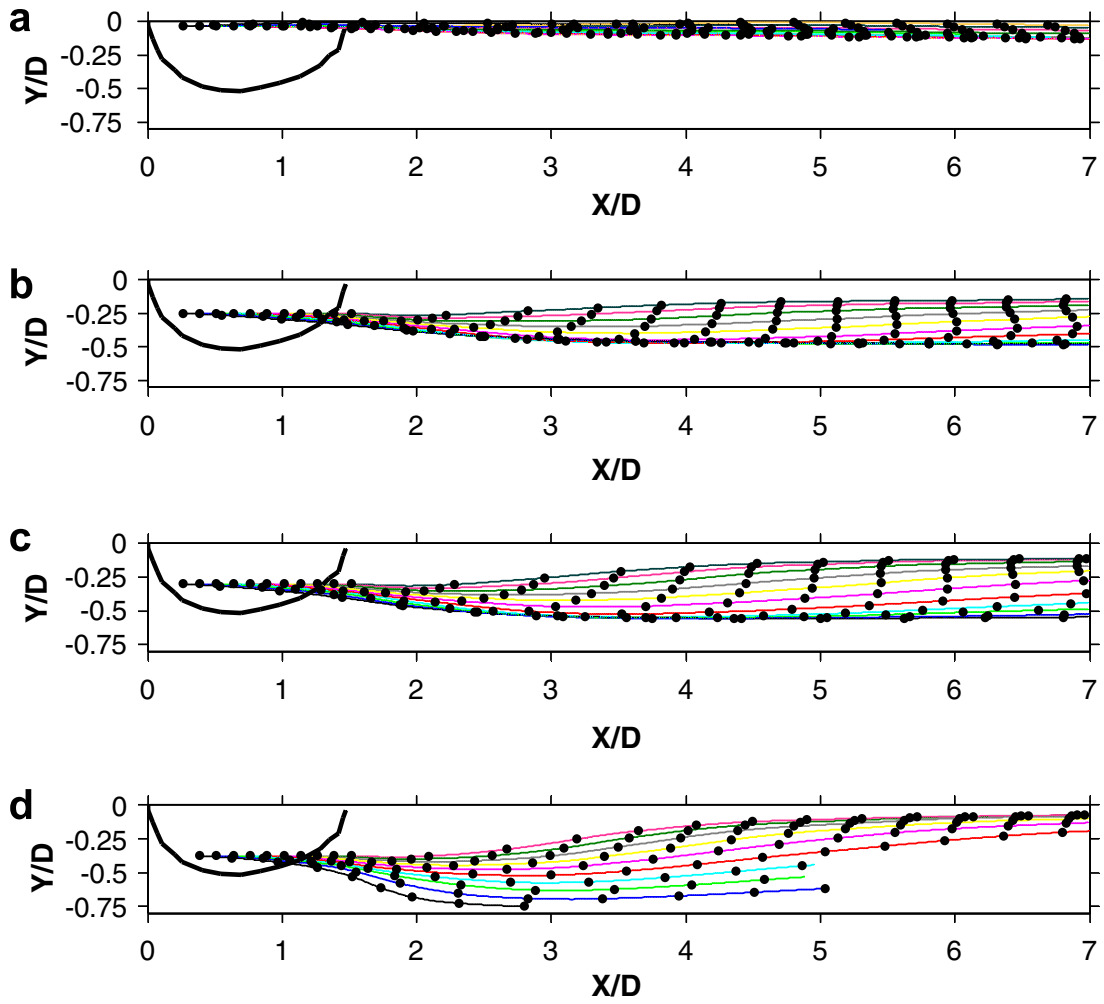

Fig. 12. Top views of streamline distributions of Fig. 11 starting from $Z / D=0.19$ with equal spacing of $0.1 \mathrm{D}$ along the streamwise direction at different transverse locations: (a) $Y / D=-0.025$, (b) $Y / D=-0.25$, (c) $Y / D=-0.30$, and (d) $Y / D=-0.375$. i.e. each dot distance along a streamline keeps a fixed time-period.

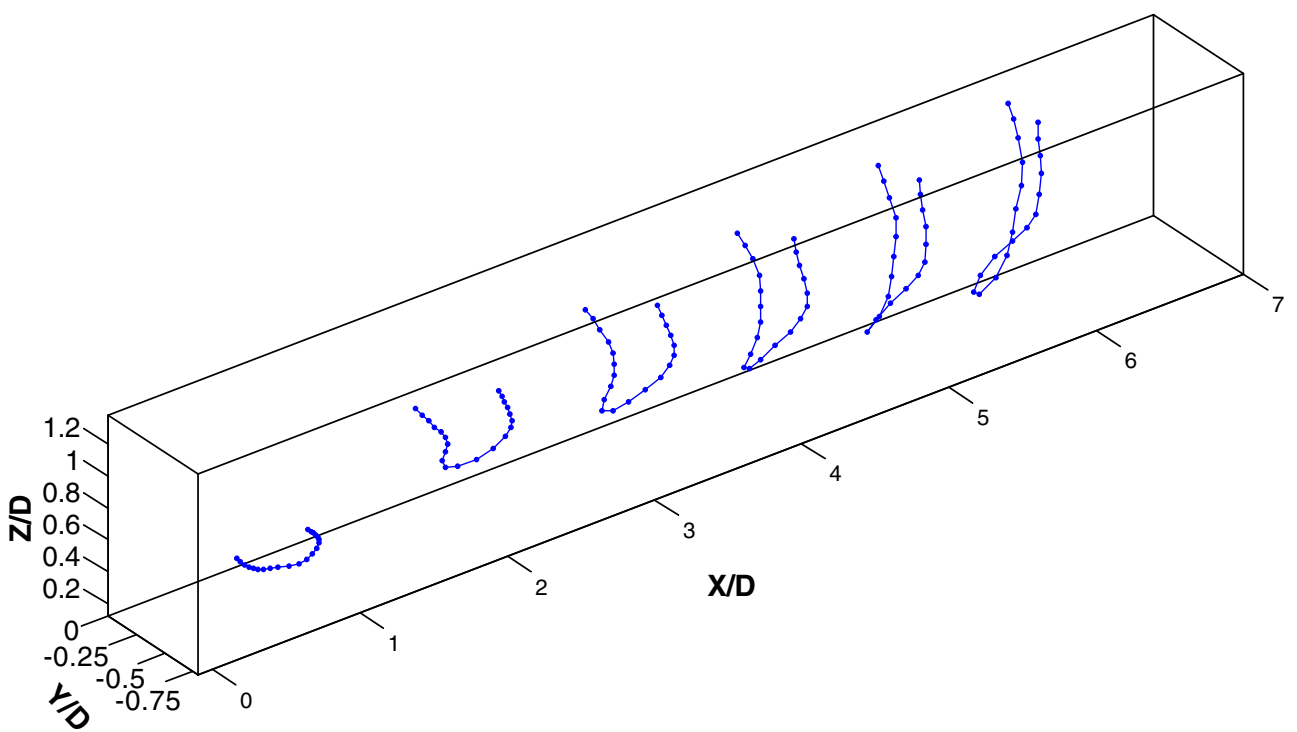

Fig. 13. Spatial evolution of a vortex ring starting directly above the injection hole.

3. Depending on flow characteristics, the ejected jet flow at transverse locations can be categorized into three flow zones, namely, a straight flow zone, a swirling flow zone, and a touch-down flow zone.
4. In addition, a ring-shape fluid filament surrounding the jet right above the hole exit is significantly stretched and bent transversely towards the wall as the ring-shape fluid filament travels downstream. 


\section{References}

[1] R.M. Kelso, T.T. Lim, A.E. Perry, An experimental study of round jets in cross-flow, J. Fluid Mech. 306 (1996) 111-144.

[2] S.H. Smith, M.G. Mungal, Mixing, structure and scaling of the jet in crossflow, J. Fluid Mech. 357 (1998) 83-122.

[3] L. Cortelezzi, A.R. Karagozian, On the formation of the counterrotating vortex pair in transverse jets, J. Fluid Mech. 446 (2001) $347-$ 373.

[4] S.D. Peterson, M.W. Plesniak, Evolution of jets emanating from short holes into crossflow, J. Fluid Mech. 503 (2004) 57-91.

[5] A. Krothapalli, L. Lourenco, J.M. Buchlin, Separated flow upstream of a jet in a crossflow, AIAA J. 28 (1990) 414-420.

[6] T.F. Fric, A. Roshko, Vortical structure in the wake of a transverse jet, J. Fluid Mech. 279 (1994) 1-47.

[7] D.S. Liscinsky, B. Truc, J.D. Holdman, Crossflow mixing of noncircular jets, J. Propuls. Power 12 (2) (1996) 225-230.

[8] S.W. Burd, R.W. Kaszeta, T.W. Simon, Measurements in film cooling flows: hole $L / D$ and turbulence intensity effects, ASME J. Turbomach. 120 (1998) 791-798.

[9] K. Thole, M. Gritsch, A. Schulz, S. Wittig, Flowfield measurement for film cooling holes with expanded exits, ASME J. Turbomach. 120 (1998) 327-336.
[10] G.M. Carlomagno, Colours in a complex fluid flow, Opt. Laser Technol. 38 (2006) 230-242.

[11] R.J. Goldstein, L.D. Stone, Row-of-holes film cooling of curved walls at low injection angles, ASME J. Turbomach. 119 (1997) 574-579.

[12] A. Melling, Tracer particles and seeding for particle image velocimetry, Meas. Sci. Technol. 8 (1997) 1406-1416.

[13] R.C. Flagan, J.H. Seinfeld, Fundamental of air pollution engineering, Prentice Hall, Englewood Cliffs, New Jersey, 1998, pp. 290-357.

[14] M. Raffel, C. Willert, J. Kompenhans, Particle image velocimetry: a practical guide, Springer, 1998, pp. 13-16.

[15] S.J. Kline, F.A. Mcclintock, Describing uncertainties in single sample experiments, Mech. Eng. (1953) 3-8.

[16] M.P. Schultz, R.J. Volino, Effects of concave curvature on boundary layer transition under high free stream turbulence conditions, ASME J. Fluid Eng. 125 (2003) 18-27.

[17] K.C. Muck, P.H. Hoffmann, P. Bradshaw, The effect of convex surface curvature on turbulent boundary layers, J. Fluid Mech. 161 (1985) 347-369.

[18] K. Karamcheti, Principles of Ideal-Fluid Aerodynamics, Stanford University, 1966 (Chapter 4, pp.162-163). 